\title{
Intervenciones poblacionales no farmacológicas en la pandemia por SARS-CoV-2. Revisión de estrategias posibles.
}

\author{
Non-pharmacological population-based interventions in \\ the SARS-CoV-2 pandemic. \\ Review of possible strategies.
}

\author{
Lucas F. de Candia, Valeria B. Bulla. \\ Carrera de Especialización en Medicina General y Familiar. Facultad de Ciencias Médicas. \\ Universidad Nacional de Rosario. Rosario; Argentina. \\ Autor por correspondencia: Lucas F. de Candia - lucasdecandia@gmail.com \\ Conflicto de intereses: no presenta.
}

\begin{abstract}
Resumen
A partir del advenimiento de la pandemia por el nuevo virus SARS-CoV-2 y de la escasa evidencia científica respecto de tratamientos farmacológicos y de vacunas para campañas masivas, las medidas poblacionales se convirtieron en herramientas fundamentales. Las distintas modalidades de implementación de intervenciones poblacionales no farmacológicas definen estrategias que han sido propuestas, proyectadas en modelos matemáticos y/o utilizadas en diferentes puntos del planeta. Se realizó una revisión narrativa de las diferentes estrategias, sus fundamentos, sus características y algunos antecedentes de utilización con el objetivo de construir un insumo para las autoridades gubernamentales en la toma de decisiones respecto del afrontamiento de la actual pandemia.
\end{abstract}

Palabras: COVID19. Pandemia. SARS-CoV-2. Confinamiento. Distanciamiento social. Aislamiento.

\begin{abstract}
With the advent of the pandemic caused by the new SARS-CoV-2 virus and the scant scientific evidence regarding pharmacological treatments and vaccines for massive campaigns, population measures became fundamental tools. The different modalities of implementation of non-pharmacological population interventions define strategies that have been proposed, projected in mathematical models and / or implemented in different places around the wolrd. A narrative review of the different strategies, their grounds, their characteristics and some background of usage was carried out with the aim of providing an input for government authorities in decision-making in terms of dealing with the current pandemic.
\end{abstract}

Key words: COVID19. Pandemic. SARS-CoV-2. Confinement. Social distancing. Isolation. 


\section{Introducción}

El nuevo virus denominado SARS-CoV-2, reportado el 31 de diciembre de 2019 en China, dio origen a una pandemia de gran impacto a nivel internacional. El reporte inicial surge a partir de casos inusuales de neumonía. En febrero de 2020, la Organización Mundial de la Salud (OMS) define la enfermedad producida por el nuevo virus como COVID19. Se conocen dos antecedentes recientes de epidemias por coronavirus: a) SARS-CoV (2003): también iniciado en China, que generó una epidemia en varios países con aproximadamente 8000 casos y 800 muertes; y b) MERS-CoV (2015): que inició en Arabia Saudita y del que se registraron aproximadamente 2500 casos y 800 muertes, con la ocurrencia ocasional de algunos casos esporádicos $(1,2,3)$.

El espectro clínico de la enfermedad por COVID-19 varía desde formas asintomáticas hasta condiciones clínicas caracterizadas por fallo respiratorio, requerimiento de atención en unidades de cuidadosintensivos(UCI), fallo multi-orgánico, sepsisy shockséptico. Unadelas primeraspublicaciones sobre esta nueva enfermedad describía un cuadro clínico caracterizado por fiebre, disnea, tos seca y malestar general. El estudio se realizó con 41 pacientes hospitalizados con confirmación de infección por SARS-CoV-2. Fiebre y tos fueron los síntomas más frecuentes: 98\% y $76 \%$, respectivamente. Todos los pacientes presentaban en la tomografía axial computarizada (TAC) de tórax signos de neumonía (4). El 11 de marzo de 2020, la OMS declaró la pandemia con más de 820 mil casos y más de 40 mil muertes asociadas a infección por SARS-CoV-2 en poco más de dos meses desde el reporte de los primeros casos. Para el primero de abril los países con más casos eran Estados Unidos, España, Italia y China dando cuenta de que la enfermedad se distribuye más allá de las fronteras políticas y desencadena grandes cadenas de trasmisión de contextos socioeconómicos muy diferentes (5).

El trabajo de Qun Li y col. analizó la epidemiología de los primeros 425 casos de neumonía asociada al nuevo virus hasta el 22 de enero de 2020 en Wuhan (China). En el mismo, se presenta evidencia que confirma la trasmisión humano-humano del nuevo virus, estiman un número reproductivo básico ${ }^{A}\left(R_{0}\right)$ de 2.2 y una duplicación de los casos cada 7.4 días. Ello sugiere que cada persona infectada transmitió la enfermedad a otras 2.2. Concluyen que se van a requerir esfuerzos considerables en el resto del mundo para reducir la trasmisión viral y controlar la epidemia (6).

En otro trabajo que analiza la epidemiología del virus a partir de los reportes de casos en China, se estimó una media de $\mathrm{R}_{0}$ de 5.7 y también concluyen en la necesidad de implementación precoz de medidas de detección y aislamiento de casos y contactos, por un lado, y de distanciamiento social, por el otro (7).

En el mes de abril, investigando clústeres de casos en Singapur se reportó evidencia que sustenta la transmisión pre sintomática del SARS-CoV-2: personas infectados pueden diseminar el virus sin síntomas o antes del desarrollo del cuadro clínico (8).

En la actualidad, el manejo de personas con COVID-19 está centrado en la detección y aislamiento de casos y contactos, la hospitalización y el tratamiento de soporte en cuadros severos. A lo largo del 2020, se han realizado una enorme cantidad de estudios científicos para evaluar eficacia y seguridad de agentes farmacológicos como antivirales, inmunomoduladores y adyuvantes. No hay aun evidencia suficiente en calidad y cantidad para aceptar ninguna terapia para su uso clínica masivo $(9,10,11,12)$. En Argentina, el Ministerio de Salud recomienda hospitalización, tratamientos de sostén, antibioticoterapia en individuos con sospecha de neumonía y glucocorticoides (GCTC) en casos severos (13). La indicación de los GCTC se basa en evidencia científica que asocia el uso de dexametasona con reducción de la mortalidad en individuos hospitalizados con compromiso respiratorio severo (14). Recientemente, en Estados Unidos la Food and Drug Administration (FDA) aprobó el Remdesivir para cuadros severos hospitalizados de COVID-19. Esta droga presenta evidencia prometedora dado que se ha observado en estudios clínicos controlados una disminución de mortalidad asociada a Remdesivir, aunque la certeza de este beneficio es baja. En el marco del estudio SOLIDARITY de la OMS no se pudo demostrar eficacia con ninguna droga de las evaluadas, una de las cuales era el Remdesivir $(15,16,17,18)$.

En relación a las vacunas, la OMS comunicó en septiembre 2020 que 36 vacunas se encuentran en fases de investigación clínica. Si bien las perspectivas son esperanzadoras, la anhelada inmunidad de rebaño por vacunas se presenta aun lejana y con algunas incertidumbres $(19,20,21)$.

A Número reproductivo básico $\left(\mathrm{R}_{0}\right)$ : es el número de casos secundarios que podría producir un caso en una población susceptible. Esta determinado por la duración del período infeccioso, la probabilidad de infectar a un individuo susceptible durante un contacto y el número de nuevos individuos susceptibles contactados por unidad de tiempo. (Ridenhour, B., Kowalik, J. M., \& Shay, D. K. (2014). Unraveling R0: considerations for public health applications. American Journal of Public Health, 104(2), e32-e41) 
En síntesis, actualmente no disponemos de suficiente evidencia científica de calidad para sustentar la recomendación de uso clínico de medidas farmacológicas, a excepción de los GCTC en cuadros severos. Por otro lado, se estima que el costo humano de la no intervención estatal en la pandemia sería demasiado alto. En consecuencia, los organismos del campo de la salud y los diferentes gobiernos, en un escenario marcado por la escasez de certezas biomédicas, recomendaron e implementaron diferentes medidas poblacionales no farmacológicas como estrategia para cuidar la vida durante el curso de la actual pandemia.

\section{¿Por qué la inmunidad de rebaño por vía natural no es una opción recomendable?}

Un individuo puede adquirir inmunidad a través de dos caminos: por vía natural (infección por el microorganismo) o mediante vacunas. La inmunidad de grupo o de rebaño se alcanza cuando en una población existe suficiente cantidad de individuos resistentes a la infección para frenar su propagación. Esta situación ofrece protección indirecta a las personas sin inmunidady, por consiguiente, susceptibles a enfermar. Se denomina "umbral de inmunidad de rebaño" al punto en el cuál la proporción de individuos susceptibles de una comunidad cae por debajo del nivel necesario para que la transmisión ocurra. En el caso de la actual pandemia, se estima que se requiere que el $67 \%$ de la población tenga inmunidad. En otras palabras, 7 de cada 10 personas en una dada población deberían ser inmunes al virus para alcanzar la inmunidad de rebaño (22).

El controversial abordaje de inmunidad de rebaño por vía natural como estrategia de afrontamiento de la pandemia por SARS-CoV-2 es una propuesta criticable, sin sustento científico y con altísimo costo humano. Básicamente, supondría la liberación de una trasmisión viral descontrolada Aun, implementando aislamiento de los grupos de alto riesgo, este abordaje implicaría graves consecuencias $(23,24)$.

Respecto de la mortalidad, se han realizado estimaciones que muestran que las pérdidas de vidas humanas se contarían por millones antes de alcanzar la inmunidad de rebaño por vía natural. Por otro lado, si bien esta descripto que la mortalidad es francamente superior en individuos de 60 o más años, de ninguna manera puede despreciarse el número de muertes registradas en otras franjas etarias. La tasa de muertes/total infectados es preocupante y se observa que a mayor número de contagios mayor número de muertes, aun en grupos de bajo riesgo (25). En el noveno reporte del Imperial College COVID-19 Response Team(26), se presentan las potenciales consecuencias de escenarios sin intervenciones en Estados Unidos (EEUU) y en Reino Unido (RU) proyectadas hasta noviembre 2021. Hicieron una predicción, por modelos matemáticos, de 2.2 millones de muertes para EEUU y de 510.000 muertes para RU. Ambos países realizaron alguna intervención poblacional: RU con bastante coordinación central, EEUU delegando la responsabilidad a las autoridades de cada Estado. Al día 20/10/20, GB llevaba registradas 44.057 muertes y EEUU, 220.824 muertes (27).

Una segunda consecuencia sería el colapso sanitario: cuanto mayor sea la trasmisión, mayor será la carga de trabajo al sistema de salud y las chances de ausencias por enfermedad en los equipos de trabajo. La saturación de los sistemas de salud puede disparar lo que se denomina "muertes indirectas" o "colaterales": aquellas no directamente vinculadas a la causa de la crisis sanitaria. Como ejemplo, la epidemia por el virus Ébola evidenció las consecuencias de la saturación del sistema en países con recursos limitados. Un estudio sobre la mortalidad indirecta en Sierra Leona en contexto de epidemia por Ébola concluyó que las muertes por otras causas fueron al menos tan importantes en número como las directamente vinculadas a la crisis sanitaria de la epidemia (28). Por otro lado, el personal sanitario, tensionado al extremo en fases de elevado número de casos/día, muestra alarmantes tasas de contagios y muertes $(29,30)$.

Además, es necesario destacar la morbilidad. Se ha descripto el COVID prolongado, como forma de la enfermedad que presenta una duración mayor de la sintomatología (31,32). También, se han reportado posibles secuelas post COVID neurológicas, cardiológicas y respiratorias cuyo impacto clínico a mediano y largo plazo aún no está del todo claro $(33,34,35)$.

Si los argumentos esbozados no fueran suficientes, se puede adicionar que no se dispone de evidencia contundente para asegurar que la infección natural por el SARS-CoV-2 genera inmunidad suficiente y perdurable.(36) Ya existen reportes de re infecciones que sugieren que la infección no garantiza el desarrollo de inmunidad protectora (37). Por consiguiente, la no intervención poblacional, permitiendo la transmisión descontrolada del virus, podría asimismo generar regiones con epidemias recurrentes y exportadoras de casos a localidades aledañas. 


\section{Medidas clásicas de salud pública para afrontamiento de epidemias}

La abrupta evolución de la pandemia por el novedoso virus agendó a la necesidad de desarrollar estrategias de abordaje mientras se carece de evidencia científica fuerte para avalar uso de tratamientos farmacológicos o vacunas. Por lo tanto, las herramientas clásicas de la salud pública asumieron un rol protagónico (38).

Tabla 1. Medidas clásicas de Salud Pública para abordaje de epidemias (adaptado de WilderSmith y Freedman, 2020)

\begin{tabular}{|c|c|c|c|c|c|}
\hline & Aplicada a & Objetivo & Características & Ventajas & Desventajas \\
\hline Aislamiento & $\begin{array}{l}\text { Personas } \\
\text { enfermas. }\end{array}$ & $\begin{array}{l}\text { Prevenir } \\
\text { contagio a } \\
\text { personas } \\
\text { sanas. }\end{array}$ & $\begin{array}{l}\text { Se ingresa a } \\
\text { la persona } \\
\text { infectada en sala } \\
\text { especialmente } \\
\text { preparadas } \\
\text { en hospitales } \\
\text { o centros de } \\
\text { aislamiento. }\end{array}$ & $\begin{array}{l}\text { Efectivo en } \\
\text { enfermedades } \\
\text { infecciosas con } \\
\text { transmisión } \\
\text { persona- } \\
\text { persona en } \\
\text { donde el } \\
\text { contagio se } \\
\text { da en la fase } \\
\text { sintomática de } \\
\text { la enfermedad. }\end{array}$ & $\begin{array}{l}\text { Poco útil cuando } \\
\text { la transmisión } \\
\text { ocurre en fases } \\
\text { pre sintomáticas } \\
\text { de la enfermedad } \\
\text { o en personas } \\
\text { asintomáticas. }\end{array}$ \\
\hline Cuarentena & $\begin{array}{l}\text { Personas } \\
\text { expuestas al } \\
\text { virus, pero no } \\
\text { enfermas. }\end{array}$ & $\begin{array}{l}\text { Reducir } \\
\text { posible } \\
\text { transmisión } \\
\text { de personas } \\
\text { expuestas } \\
\text { antes la } \\
\text { aparición de } \\
\text { síntomas. }\end{array}$ & $\begin{array}{l}\text { Se aísla en } \\
\text { domicilio a } \\
\text { la persona } \\
\text { expuesta. }\end{array}$ & $\begin{array}{l}\text { Interrumpe } \\
\text { cadena de } \\
\text { contagios en } \\
\text { personas que no } \\
\text { desarrollaron } \\
\text { síntomas. }\end{array}$ & $\begin{array}{l}\text { Requiere de la } \\
\text { colaboración de } \\
\text { las personas o } \\
\text { de leyes/normas } \\
\text { que obliguen al } \\
\text { cumplimiento. } \\
\text { Producen } \\
\text { importante } \\
\text { ausentismo } \\
\text { laboral. Pierde } \\
\text { eficacia en área } \\
\text { con circulación } \\
\text { comunitaria alta } \\
\text { o momentos de } \\
\text { aumento abrupto } \\
\text { de casos }\end{array}$ \\
\hline $\begin{array}{l}\text { Distanciamiento } \\
\text { social }\end{array}$ & \multirow[t]{2}{*}{$\begin{array}{l}\text { Comunidades, } \\
\text { ciudades o } \\
\text { regiones con } \\
\text { circulación } \\
\text { comunitaria } \\
\text { del virus. }\end{array}$} & \multirow[t]{2}{*}{$\begin{array}{l}\text { Prevenir } \\
\text { contacto en } \\
\text { comunidades } \\
\text { de personas } \\
\text { infectadas } \\
\text { y personas } \\
\text { sanas. }\end{array}$} & $\begin{array}{l}\text { Cancelación } \\
\text { de reuniones } \\
\text { públicas, cierre } \\
\text { de escuelas, } \\
\text { teletrabajo, } \\
\text { uso masivo de } \\
\text { máscaras faciales } \\
\text { en vía pública. }\end{array}$ & \multirow{2}{*}{$\begin{array}{l}\text { Medida efectiva } \\
\text { en localidades } \\
\text { con alta } \\
\text { transmisión } \\
\text { comunitaria } \\
\text { donde el } \\
\text { aislamiento y } \\
\text { la cuarentena } \\
\text { de casos y } \\
\text { contactos es } \\
\text { insuficiente } \\
\text { para contener } \\
\text { la epidemia. }\end{array}$} & \multirow[t]{2}{*}{$\begin{array}{l}\text { Requiere } \\
\text { colaboración } \\
\text { de las personas } \\
\text { o leyes/normas } \\
\text { que obliguen al } \\
\text { cumplimiento. } \\
\text { Impacto en } \\
\text { interrupción de } \\
\text { actividades }\end{array}$} \\
\hline $\begin{array}{l}\text { Confinamiento } \\
\text { general }\end{array}$ & & & $\begin{array}{l}\text { Prohibición de } \\
\text { toda actividad, } \\
\text { con excepción } \\
\text { de aquellas } \\
\text { definidas como } \\
\text { esenciales. }\end{array}$ & & \\
\hline
\end{tabular}

\section{¿Por qué se requieren medidas poblacionales para afrontar la actual pandemia?}

A la espera de campañas de vacunación masivas y de tratamientos farmacológicos con demostrada utilidad, las medidas poblacionales no farmacológicas se presentan como la intervención más efectiva para frenar, disminuir y enlentecer la diseminación del virus en las comunidades $(39,40)$. 
En muchos países, se optó por medidas globales de distanciamiento social (suspensión de actividades laborales y educativas) como estrategia para frenar la trasmisión viral, disminuir el número de casos y bajar la mortalidad. Si bien, dichas medidas dispararon controversias y debates de diferente índole a nivel internacional, la comunidad científica se mantuvo firme en la postura de recomendar las medidas poblacionales no farmacológicas como intervención efectiva para frenar la trasmisión y atenuar el impacto de la pandemia en términos de contagios y muertes (41,42).

Lo que se pudo registrar de la experiencia de China: las estrategias poblacionales no farmacológicas utilizadas para contener el primer brote de la enfermedad fueron efectivas (43). Las medidas implementadas pueden agruparse en tres grupos: restricciones de viajes (para evitar dispersión inter localidades del virus), identificación/aislamiento activo de casos y contactos (dispositivos de rastreo y detección) y distanciamiento social (restricciones de contacto en general, cuidados personales como el lavado de manos y el uso de mascarillas, prohibición de reuniones/actividades con acumulación de personas). Las medidas implementadas por el gobierno chino fueron agresivas, precoces y multifacéticas. La temprana implementación fue clave para evitar mayor diseminación del virus dentro y fuera del país. Tres observaciones principales pueden realizarse a partir de los resultados publicados. La primera es que la movilidad de las personas y los contactos cercanos tienen un rol fundamental en la transmisión del SARS-CoV-2. La segunda, la importancia y el efecto de las medidas no farmacológicas combinadas. La tercera, dado que ya han retomado actividades y viajes, medidas como el rastreo activo y precoz de casos y su aislamiento deben sostenerse aun luego de controlada la "primera ola" de la pandemia.

Mediante un modelo matemático utilizado en las epidemias de gripe, Koo J. y col. demostraron que en Singapur la implementación combinada de intervenciones de cuarentena de casos infectados y contactos cercanos, cierre de escuelas y distanciamiento del trabajo o teletrabajo una vez que la trasmisión comunitaria fue detectada puede reducir sustancialmente el número de infecciones por SARS-CoV-2 (44).

La revista NATURE, publicó un artículo en junio 2020 donde se investiga el efecto de las restricciones poblacionales implementadas en 11 países de Europa (45). Estiman que las intervenciones no farmacológicas combinadas se relacionan con importantes reducciones en los tiempos de duplicación del número de casos/día. Además, comparando la predicción de muertes en modelos matemáticos con y sin intervenciones, calcularon que con las medidas poblacionales implementadas se habrían evitado alrededor de 3 millones de muertes en 11 países desde el inicio de la pandemia.

Un estudio que evaluó el efecto de las recomendaciones de "quedarse en casa" y definiciones gubernamentales de distanciamiento social en los EEUU demostró que estas medidas lograron disminuir significativamente la tasa de incidencia y la mortalidad por COVID (46).

Un consenso de científicos publicó recientemente su posición en The Lancet (47) manifestando que deben ser ampliamente implementadas medidas efectivas para controlar y/o suprimir la trasmisión viral. A su vez, señalan la necesidad de que dichas medidas cuenten con soporte de programas sociales y de financiamiento para favorecer el acompañamiento de las medidas por parte de la ciudadanía y, también, para abordar las inequidades que esta pandemia profundizó en todo el mundo. También, esbozaron una contundente crítica a cualquier abordaje basado en la búsqueda de la inmunidad de rebaño por vía natural.

\section{Diferentes estrategias de intervención poblacional}

\section{Supresión de la transmisión viral}

El objetivo en esta intervención es profundo y ambicioso: suprimir la transmisión viral. Para lograrlo se requiere de la implementación de restricciones de actividades y de la movilidad de personas en un área geográfica dada y por un periodo suficiente de tiempo. En términos epidemiológicos, se necesita alcanzar un $\mathrm{R}_{0}$ menor a 1, para empujar progresivamente la curva hacia abajo, disminuyendo los números de nuevos casos (48).

La situación de Nueva Zelanda, merece ser observada. Ante el aumento de casos, el 26 de marzo el gobierno define la implementación de un confinamiento general en todo el territorio. Disparó incertidumbre al comienzo, pero, luego los contagios comenzaron a disminuir. Se completó un total de 7 semanas de "quedarse en casa" como norma nacional. En mayo el último caso detectado fue puesto en aislamiento y se declaró el fin de la transmisión comunitaria en el territorio neozelandés. Entre las reflexiones que esta experiencia ofrece, es pertinente señalar: 1- las intervenciones poblacionales rápidas y basadas en las definiciones científicas fueron clave, 2-fue efectivo combinar medidas: cierre fronteras, suspensión masiva actividades, detección 
de casos; 3- el liderazgo empático de la Primer Ministra Jacinda Ardern y la estrategia discursiva ${ }^{B}$ tuvieron efectos positivos en la convocatoria amplia de la ciudadanía a cumplir con las medidas implementadas (49).

\section{Mitigación sostenida como medida de atenuación}

Luego de amplios cierres generales (“lockdown”) que frenaron y postergaron el pico de casos, algunos países optaron por estrategias de sólo mitigación con medidas como uso general de mascarillas faciales, protocolos con distanciamiento y prohibición de actividades que impliquen acumulación de personas. Así, se pretende sostener un $\mathrm{R}_{0}$ por debajo del "natural” que se estimó en la dinámica de la epidemia sin intervenciones (entre 2 y 4) pero aceptando que no se logrará llevarlo por debajo de 1. Entre los beneficios, se pueden destacar el marcada efecto supresor de casos y muertes a partir del cierre inicial, la preparación y ampliación de capacidad del sistema de salud, la organización de dispositivos de rastreo, detección, diagnóstico, aislamiento y seguimiento de casos y contactos y la instauración de abordajes agresivos de bloqueo de "focos de contagios". Sin embargo, las aperturas post cierre total pueden generar algunas consecuencias no deseables: aumentos abruptos de casos, percepción general de la ciudadanía de que el problema fue resuelto y desarrollo de "pico" de casos con saturación del sistema de salud. Los sistemas de rastreo de casos y contactos son menos efectivos en territorios de alta densidad poblacional, en regiones con gran dispersión (pequeños pueblos muy distantes de las ciudades que concentran recursos) y en momentos de aumento masivo de casos. A su vez, la ampliación de los sistemas sanitarios tiene algunos puntos limitantes como: 1- escasa posibilidad de aumentar recursos críticos como equipos de asistencia respiratoria mecánica, 2- limitaciones en personal capacitado para tareas específicas, 3- disponibilidad acotada de equipamiento de protección personal, y 4- dificultad de infraestructura histórica en hospitales que no puede resolverse en el corto plazo (50).

En general, cuando la enfermedad supero la capacidad de contención y se dispara la circulación comunitaria, muchos países optaron por estrategias de mitigación como EEUU, España e Italia (51). Suecia adoptó una de las estrategias de mitigación más liberales del mundo sin confinamiento y con locales gastronómicos y gimnasios abiertos, mientras se alentaban normas de distanciamiento físico. En marzo, de la mano del epidemiólogo Agnes Tegnell, las autoridades desaconsejaron el uso masivo de máscaras faciales y se opusieron a las medidas de distanciamiento social implementadas por otros países. Incluso (inicialmente), delegaron en cada individuo infectado el reporte de sus contactos y no establecieron normas de obligatoriedad de cuarentena de contactos de casos confirmados. Más aún, mientras estuvieran asintomáticos eran invitados a continuar sus actividades. Se podría definir que utilizaron una de las estrategias de rastreo, detección y aislamiento menos agresiva del mundo. Sin embargo, para octubre modificaron sus protocolos e incluyeron la indicación de cuarentena domiciliaria de contactos de infectados y, progresivamente, el aumento de casos forzó la implementación de medidas poblacionales más restrictivas (52).

\section{Confinamientos regionales o zonales}

Se basan en la idea de establecer barreras entre comunidades con altos reportes de casos y aquellos sin transmisión reportada. Se han utilizado históricamente bajo la denominación de "cordones sanitarios". En la actual pandemia, se ha utilizado esta estrategia identificando, en tiempo real, zonas "rojas": brotes con clústeres de alto número de casos. A partir de precisos sistemas de información, se dividen las zonas de una región en "verdes" (pocos o nulos casos reportados) y "rojas”. En las segundas, se instauran estrictas medidas de confinamiento (53).

Francia adoptó esta estrategia: dado que el confinamiento total es complejo de sostener, se permitió la movilidad, pero estableciendo límites precisos entre áreas "verdes" y "rojas”, y definiendo restricciones rigurosas de movilidad entre áreas. De esta manera, por ejemplo, dos ciudadanos parisinos de diferentes distritos no podrían contagiarse entre sí, ni siquiera mediante contactos intermediarios. La clave de su éxito es el adecuado funcionamiento de las barreras entre áreas. Además, esta estrategia permite minimizar las derivaciones negativas sociales y económicas del confinamiento estricto, acotándolo sólo a las áreas "rojas". Su eficacia depende del funcionamiento a tiempo real de los sistemas de información y de dispositivos de rastreo y detección de casos y contactos para identificar y definir velozmente cada zona "roja". La re unificación de las zonas "verdes" se proyecta a medida

B Bajo el lema "team of 5 million" ("equipo de 5 millones"), el marco del cuidado colectivo y la salud como bien social común fue central en la estrategia discursiva de la Primer Ministra Jacinda Ardern. 
que se acumulan áreas cercanas sin reportes de casos. Factores como interdependencia social y económica entre zonas puede reducir la eficacia de esta estrategia (54).

En Inglaterra también se implementó este tipo de medidas: la ciudad de Leicester presentó amplia diferencia de casos confirmados respecto de urbes cercanas, por lo que fue foco de restricciones mayores que las sostenidas en localidades aledañas (55).

\section{Confinamientos intermitentes}

Esta estrategia implica confinamientos intermitentes que se apliquen y se levantan periódicamente. Se describe como potencialmente efectiva para controlar y disminuir el valor de $\mathrm{R}_{0} \mathrm{y}$, por consiguiente, la proporción de cuadros graves y muertes (56). Esta es una modalidad de abordaje posible y recomendable en países con localidades con alta densidad poblacional, patrones de alto contacto, dificultades económicas, sistemas de salud limitados en su capacidad de ampliación y sistemas de rastreo, detección y aislamiento acotados. Esta estrategia atiende tanto las necesidades económicas como las sanitarias. Los cierres intermitentes y planificados ofrecen: 1- posibilidad de ordenamiento de la vida social con proyecciones que pueden resultar aliviadoras subjetivamente, 2- evitar el ahogo de la economía que supone la búsqueda de la supresión viral con cierres totales prolongados, 3- frenar cadenas de contagios con la interrupción de la movilidad de muchas personas de manera intermitente, y 4- evitar el colapso sanitario y sus catastróficas consecuencias (57).

En un trabajo reciente, se realizó un modelo matemático para medir los efectos de cierres intermitentes en 16 países comparando dos esquemas: 50 días de confinamiento + 30 días de relajación vs 50 días de mitigación + 30 días de relajación. El primer esquema resultó más efectivo para reducir el $R_{0}$ por debajo de 1, prevenir la ocupación alta de camas críticas y disminuir los fallecimientos, en comparación con el segundo esquema o con el escenario supuesto de no intervención poblacional (58).

Científicos del CONIET en Argentina han propuesta una estrategia similar: Aislamiento Selectivo, Planificado e Intermitente (ASPI) $(59,60,61,62)$. Se trata de una estrategia con periodos de cierres intercalados con fases de apertura. La selectividad sería regional, temporal y por actividades. La planificación ofrecería una agenda de intermitencia preestablecida para ofrecer un panorama de certeza a la ciudadanía y para la planificación de actividades laborales, productivas y recreativas con un horizonte de corto y mediano plazo. Esta opción aleja la posibilidad de colapso sanitario. Recomiendan que debe ser complementada con una eficiente capacidad de rastreo, detección y aislamiento de casos y contactos (63). La trazabilidad de los casos, amplia y eficazmente lograda ciudades como Rosario, podría volver a ser posible (64).

\section{Discusión}

Actualmente, las principales medidas con demostrada eficacia en el afrontamiento de la actual pandemia por el nuevo virus SARS-CoV-2 son aquellas que disminuyen la posibilidad de contacto entre infectados y susceptibles. Herramientas clásicas de la salud pública como el aislamiento de casos, cuarentena de contactos, el distanciamiento social y el confinamiento vuelven a presentarse como fundamentales en el contexto presente. Hasta que se disponga de vacunas seguras y eficaces para campañas masivas y se alcance inmunidad de rebaño por vacuna, las intervenciones no farmacológicas son el único camino posible. La convocatoria a un acuerdo social y político amplio, bajo el estandarte del cuidado colectivo, parece ineludible si se pretende salvar vidas.

La inmunidad de rebaño por vía natural no es una opción ética ni aceptable. Por otro lado, las medidas generales en un país extenso y heterogéneo como Argentina no parecen ser la mejor alternativa en el futuro inmediato. El diseño de estrategias contextualizadas en las realidades particulares de cada localidad/región podría ser el camino más adecuado. En coyunturas donde la crisis económica y social no ofrece margen para la implementación de confinamientos prolongados, la supresión viral parece un objetivo inaccesible. Siguiendo las sugerencias de los científicos de CONICET, la estrategia de confinamientos breves e intermitentes con eficiente capacidad de detección y aislamiento de casos y contactos parecen ser el camino de afrontamiento de la pandemia más coherente y recomendable en Argentina. El gran desafío de las estrategias intermitentes tiene dos dimensiones: 1- la capacidad de los gobernantes de implementarlas convocando ampliamente a la ciudadanía y a todos los sectores del arco político, y 2- el diseño contextualizado de estrategias intermitentes selectivas y planificadas, contemplando miradas integrales de las necesidades de cada territorio, priorizando los sectores más fragilizados por la pandemia. 
La experiencia de países como Nueva Zelanda merece ser observada. Más allá de los detalles técnicos de la estrategia implementada, es necesario destacar el llamamiento al acuerdo social y político. El claro mensaje del cuidado colectivo como eje fundamental.

Fuente de financiamiento: no presenta.

\section{Referencias bibliograficas}

1. Cascella M, Rajnik M, Cuomo A, et al. Features, Evaluation, and Treatment of Coronavirus. [Updated 2020 Oct 4]. In: StatPearls [Internet]. Treasure Island (FL): StatPearls Publishing; 2020 Jan-. Available from: https://www.ncbi.nlm.nih.gov/books/NBK554776/

2. Cheng VC, Lau SK, Woo PC, et al. Severe acute respiratory syndrome coronavirus as an agent of emerging and reemerging infection. Clin Microbiol Rev. 2007;20(4):660-694.

3. Chan JF, Lau SK, To KK, et al. Middle East respiratory syndrome coronavirus: another zoonotic betacoronavirus causing SARS-like disease. Clin Microbiol Rev. 2015;28(2):465-522.

4. Huang C, Wang Y, Li X, et al. Clinical features of patients infected with 2019 novel coronavirus in Wuhan, China. Lancet. 2020 Feb 15;395(10223):497-506.

5. World Health Organization. Coronavirus disease (COVID-2019): situation report 72 [Internet].

Genebra: World Health Organization; 2020. Disponible en: https:/www.who.int/docs/default-source/coronaviruse/situation-reports/20200401-sitrep-72-covid-19.pdf?sfvrsn=3dd8971b_2

6. Li Q, Guan X, Wu P, et al. Early Transmission Dynamics in Wuhan, China, of Novel Coronavirus-Infected Pneumonia. N Engl J Med. 2020 Mar 26;382(13):1199-1207.

7. Sanche S, Lin Y, Xu C. High Contagiousness and Rapid Spread of Severe Acute Respiratory Syndrome Coronavirus 2. Emerging Infectious Diseases. 2020; 26(7), 1470-1477.

8. Wei WE, Li Z, Chiew CJ. Presymptomatic Transmission of SARS-CoV-2-Singapore, January 23-March 16, 2020. Morbidity and Mortality Weekly Report. 2020; 69(14), 411.

9. Lam S, Lombardi A, Ouanounou A. COVID-19: A review of the proposed pharmacological treatments. European journal of pharmacology. 2020; 886, 173451.

10. McCreary EK, Pogue JM. Coronavirus Disease 2019 Treatment: A Review of Early and Emerging Options. Open Forum Infect Dis. 2020 Mar 23;7(4):ofaal05.

11. Bhimraj A, Morgan RL, Shumaker AH, et al. Infectious Diseases Society of America Guidelines on the Treatment and Management of Patients with COVID-19. Clin Infect Dis. 2020 Apr 27:ciaa478.

12. COVID-19 Treatment Guidelines Panel. Coronavirus Disease 2019 (COVID-19) Treatment Guidelines. National Institutes of Health. Available at https://www.covid19treatmentguidelines.nih.gov/. Accessed [01/12/20].

13. Ministerio de Salud de la Argentina. Recomendaciones condicionales para el abordaje terapéutico de COVID-19 - Versión 3.0 (septiembre 2020). Disponible en: https://www.argentina.gob.ar/salud/coronavirus-COVID-19/abordaje-terapeutico. Accceso 01/12/20

14. Horby P, Lim WS, Emberson JR, et al. Dexamethasone in Hospitalized Patients with Covid-19 - Preliminary Report. N Engl J Med. 2020 Jul 17:NEJMoa2021436.

15. Beigel JH, Tomashek KM, Dodd LE. Remdesivir for the Treatment of Covid-19—Final Report. N Engl J Med 2020; 383:1813-1826.

16. Wang Y, Zhang D, Du G, et al. Remdesivir in adults with severe COVID-19: a randomised, double-blind, placebo-controlled, multicentre trial. Lancet. 2020;395(10236):1569-1578.

17. Dolin R, Hirsch MS. Remdesivir—An Important First Step. N Engl J Med 2020; 383:1886-1887

18. WHO Solidarity Trial Consortium. (2020). Repurposed antiviral drugs for COVID-19-interim WHO SOLIDARITY trial results. New England Journal of Medicine.

19. Hodgson SH, Mansatta K, Mallett G, et al. What defines an efficacious COVID-19 vaccine? A review of the challenges assessing the clinical efficacy of vaccines against SARS-CoV-2. Lancet Infect Dis. 2020 Oct 27:S1473-3099(20)30773-8.

20. Dong Y, Dai T, Wei Y, et al. A systematic review of SARS-CoV-2 vaccine candidates. Signal transduction and targeted therapy 5.1 (2020): 1-14.

21. Heaton PM. The Covid-19 vaccine-development multiverse. N Engl J Med 2020; 383:1986-1988

22. Randolph HE, Barreiro LB. Herd Immunity: Understanding COVID-19. Immunit 2020, 52(5), 737-741.

23. Alwan NA, Burgess RA, Ashworth S, et al. Scientific consensus on the COVID-19 pandemic: we need to act now. The Lancet 2020, 396(10260), e71-e72.

24. Fontanet, A, Cauchemez S. COVID-19 herd immunity: where are we?. Nature Reviews Immunology 2020, 20(10), 583-584.

25. Verity R, Okell LC, Dorigatti I, et al. Estimates of the severity of coronavirus disease 2019: a model-based analysis. Lancet Infect Dis 2020; 20: 669-77.

26. Ferguson N, Laydon D, Nedjati-Gilani G, et al. Report 9: Impact of non-pharmaceutical interventions (NPIs) to reduce COVID19 mortality and healthcare demand. Imperial College London 2020, 10, 77482

27. Coronavirus Source Center. John Hopkins University. Disponible en: https://coronavirus.jhu.edu/ (Acceso 20/10/20)

28. Sochas L, Channon AA, Nam S. Counting indirect crisis-related deaths in the context of a low-resilience Health system: the case of maternal and neonatal health during the Ebola epidemic in Sierra Leone. Health Policy Plan 2017; 32(suppl_3): iii32-iii39

29. Wang J, Zhou M, Liu F. Reasons for healthcare workers becoming infected with novel coronavirus disease 2019 (COVID-19) in China. J Hosp infect 2020, 105(1).

30. Lapolla P, Mingoli A, Lee R. Deaths from COVID-19 in healthcare workers in Italy —What can we learn?. Infection Control \& Hospital Epidemiology 2020, 1-2. 
31. Nature. Long COVID: let patients help define long-lasting COVID symptoms. Nature 2020; 586: 170.

32. Carfi A, Bernabei R, Landi F. Persistent symptoms in patients after acute COVID-19. Jama 2020, 324(6), 603-605.

33. Huang L, Zhao P, Tang D, et al. Cardiac Involvement in Patients Recovered From COVID-2019 Identified Using Magnetic Resonance Imaging. JACC Cardiovasc Imaging. 2020;13(11):2330-2339.

34. Mitrani RD, Dabas N, Goldberger JJ. COVID-19 cardiac injury: Implications for long-term surveillance and outcomes in survivors. Heart Rhythm. 2020;17(11):1984-1990.

35. Lahiri D, Ardila A. COVID-19 Pandemic: A Neurological Perspective. Cureus. 2020;12(4):e7889. Published 2020 Apr 29.

36. Chen Y, Tong X, Li Y, et al. A comprehensive, longitudinal analysis of humoral responses specific to four recombinant antigens of SARS-CoV-2 in severe and non-severe COVID-19 patients. PLoS Pathogens 2020, 16(9), el008796.

37. Parry J. COVID-19: Hong Kong scientists report first confirmed case of reinfection. BMJ 2020; 370: m3340

38. Wilder-Smith A, Freedman DO. Isolation, quarantine, social distancing and community containment: pivotal role for old-style public health measures in the novel coronavirus (2019-nCoV) outbreak. Journal of travel medicine 2020, 27(2), taaa020.

39. Delen D, Eryarsoy E, Davazdahemami B. No Place Like Home: Cross-National Data Analysis of the Efficacy of Social Distancing During the COVID-19 Pandemic. JMIR public health and surveillance 2020, 6(2), e19862.

40. Flaxman S, Mishra S, Gandy A, et al. Estimating the effects of non-pharmaceutical interventions on COVID-19 in Europe. Nature 2020; 584: 257-61.

41. Parmet WE, Sinha MS. Covid-19—-the law and limits of quarantine. New England Journal of Medicine 2020, 382(15), e28.

42. Lewnard JA, Lo NC. Scientific and ethical basis for social-distancing interventions against COVID-19. Lancet Infect Dis. 2020;20(6):631-633.

43. Lai S, Ruktanonchai NW, Zhou L, et al. Effect of non-pharmaceutical interventions to contain COVID-19 in China. Nature. 2020 Sep;585(7825):410-413.

44. Koo JR, Cook AR, Park M, et al. Interventions to mitigate early spread of SARS-CoV-2 in Singapore: a modelling study [published correction appears in Lancet Infect Dis. 2020 May;20(5):e79]. Lancet Infect Dis. 2020;20(6):678-688.

45. Flaxman S, Mishra S, Gandy A, et al. Estimating the effects of non-pharmaceutical interventions on COVID-19 in Europe. Nature 2020, 584(7820), 257-261.

46. VoPham T, Weaver MD, Hart JE, Ton M, White E, Newcomb PA. Effect of social distancing on COVID-19 incidence and mortality in the US. Preprint. medRxiv. 2020;2020.06.10.20127589.

47. Alwan NA, Burgess RA, Ashworth S, et al. Scientific consensus on the COVID-19 pandemic: we need to act now [published correction appears in Lancet. 2020 Oct 19;:]. Lancet. 2020;396(10260):e71-e72.

48. Ferguson N, Laydon D, Nedjati-Gilani G, et al. Report 9: Impact of non-pharmaceutical interventions (NPIs) to reduce COVID19 mortality and healthcare demand. Imperial College London 2020, 10, 77482

49. Baker MG, Wilson N, Anglemyer A. Successful elimination of Covid-19 transmission in New Zealand. New England Journal of Medicine 2020, $383(8)$, e56.

50. Chowdhury R, Luhar S, Khan N, et al. Long-term strategies to control COVID-19 in low and middle-income countries: an options overview of community-based, non-pharmacological interventions. European journal of epidemiology 2020, 35(8), 743-748.

51. Walensky RP, Del Rio C. From Mitigation to Containment of the COVID-19 Pandemic: Putting the SARS-CoV-2 Genie Back in the Bottle. JAMA. 2020;323(19):1889-1890.

52. Gretchen V. Sweden's gamble. Science 2020, 370 (6513), 159-163.

53. Chowdhury R, Luhar S, Khan N, et al. Long-term strategies to control COVID-19 in low and middle-income countries: an options overview of community-based, non-pharmacological interventions. European journal of epidemiology 2020, 35(8), 743-748.

54. Chowdhury R, Luhar S, Khan N, et al. Long-term strategies to control COVID-19 in low and middle-income countries: an options overview of community-based, non-pharmacological interventions. European journal of epidemiology 2020, 35(8), 743-748.

55. Mahase E. Covid-19: How does local lockdown work, and is it effective?. BMJ. 2020;370:m2679. Published 2020 Jul 3.

56. Ferguson N, Laydon D, Nedjati-Gilani G, et al. Report 9: Impact of non-pharmaceutical interventions (NPIs) to reduce COVID19 mortality and healthcare demand. Imperial College London 2020, 10, 77482.

57. Chowdhury, R. (2020). Rolling lockdowns could protect both economies and health in low-income countries. The Conversation. (02/06/20) Disponible en: https://theconversation.com/rolling-lockdowns-could-protect-both-economies-and-health-in-low-income-countries-139054

58. Chowdhury R, Heng K, Shawon MSR, et al. Dynamic interventions to control COVID-19 pandemic: a multivariate prediction modelling study comparing 16 worldwide countries. European journal of epidemiology 2020, 35(5), 389-399.

59. Botón rojo y aislamiento intermitente. Ernesto Kofman (Encuentro Virtual, 17/10/20) Disponible en: https://www.youtube.com/watch?$\mathrm{v}=$ Oex15mhvzQg\&feature=youtu.be

60. Botón rojo y aislamiento intermitente. Rodrigo Castro (Encuentro Virtual, 17/10/20) Disponible en: https://www.youtube.com/watch?v=jOM61IekQ30\&feature=youtu.be

61. Un llamado para que el Estado sostenga el aislamiento y ayude a los más vulnerables (Nota periodística, 12/8/20) Disponible en: https://www. paginal2.com.ar/284430-un-llamado-para-que-el-estado-sostenga-el-aislamiento-y-ayud

62. Dr. Rodrigo Castro: “Aislamiento, selectivo, planificado e intermitente es nuestra propuesta” (Encuentro Virtual, 17/10/20) Disponible en: https:// www.youtube.com/watch?v=VK_w5lnfpWo

63. Alberto Kornblihtt: “No podemos naturalizar el crecimiento exponencial de muertes que tenemos" (Nota periodística, 27/10/20) Disponible en: https://www.paginal2.com.ar/290336-alberto-kornblihtt-no-podemos-naturalizar-el-crecimiento-exp

64. Kofman describió los escenarios posibles de pandemia en Rosario y analizó cómo bajar los casos. (Nota periodística, 27/10/20) Disponible en: https:// www.elciudadanoweb.com/kofman-describio-los-escenarios-posibles-de-pandemia-en-rosario-y-analizo-como-bajar-los-casos/ 